\title{
Multiple Olfactory Receptor Neurons and Their Axonal Projections in the Antennal Lobe of the Honeybee Apis mellifera
}

\author{
CHRISTINA KELBER, WOLFGANG RÖSSLER, \\ AND CHRISTOPH JOHANNES KLEINEIDAM* \\ Department of Behavioral Physiology and Sociobiology, Biozentrum, \\ University of Würzburg, 97074 Würzburg, Germany
}

\begin{abstract}
The poreplate sensilla of honeybees are equipped with multiple olfactory receptor neurons (ORNs), which innervate glomeruli of the antennal lobe (AL). We investigated the axonal projection pattern in glomeruli of the AL (glomerular pattern), formed by the multiple ORNs of individual poreplate sensilla. We used the different glomerular patterns to draw conclusions about the equipment of poreplate sensilla with different ORN types. ORNs of single poreplate sensilla were stained and analyzed by laser-scanning confocal microscopy and 3D software (AMIRA). In 13 specimens we found between 7 and 23 ORNs. This is in accordance with data found in the literature (5-35 ORNs) suggesting that all ORNs of the single poreplate sensilla were stained. The ORNs innervate the AL via all four sensory tracts (T1-T4), and glomeruli of the anterior part of the AL are more often innervated. Each ORN innervates a single glomerulus (uniglomerular), and all ORNs of one poreplate sensillum project to different glomeruli. Visual inspection and individual identification of glomeruli, based on the honeybee digital AL atlas, were used to evaluate mapping of glomeruli by a rigid transformation of the experimental ALs onto a reference AL. ORNs belonging to individual poreplate sensilla form variable glomerular patterns, and we did not find a common organization of glomerular patterns. We conclude that poreplate sensilla are equipped with different ORN types but that the same ORN types can be found in different poreplate sensilla. The equipment of poreplate sensilla with ORNs is overlapping. The mapping of glomeruli by rigid transformation is revealed to be a powerful tool for comparative neuroanatomy.
\end{abstract}

Indexing terms: sensilla placodea; 3D reconstruction; antennal lobe; odor representation; VOI; glomeruli

In many cases, animal behavior is guided by olfaction, and most animals have developed sensory organs and olfactory receptor neurons (ORNs) with remarkable sensitivity to detect and discriminate a vast diversity of different odors. The interface for detecting odors in the environment in vertebrates is the nasal mucosa, with its embedded dendrites of ORNs; in insects the interface is the antenna, with numerous olfactory sensilla and associated ORNs. ORN axons project to the first olfactory neuropil and terminate in glomeruli of the vertebrate olfactory bulb $(\mathrm{OB})$ and the insect antennal lobe $(\mathrm{AL})$. The glomeruli represent functional units of the first olfactory neuropil that receive input from ORNs such that a chemotopic representation emerges. The first olfactory neu- ropil shares many common design principles across phyla (Hildebrand and Shepherd, 1997; Strausfeld and Hildebrand, 1999).

Grant sponsor: Deutsche Forschungsgemeinschaft; Grant number: SFB 554 (A6); Grant sponsor: University of Würzburg.

*Correspondence to: Christoph Johannes Kleineidam, Department of Behavioral Physiology and Sociobiology, Biozentrum, University of Würzburg, Am Hubland, 97074 Würzburg, Germany.

E-mail: kleineidam@biozentrum.uni-wuerzburg.de 
For this and several other, more practical, reasons, selected insect species have proved to be good model systems to study olfaction. Comparative neuroanatomical studies in insects revealed that the arrangement of glomeruli in the AL is highly organized. The AL design is species specific (Rospars, 1988; Vickers et al., 1998; Berg et al., 2005; Kleineidam et al., 2005), and functionally corresponding glomeruli are almost invariant in their relative position across individuals (Joerges et al., 1997; Galizia et al., 1999b; Sachse et al., 1999). With the aid of confocal laser-scanning microscopy and computer-aided data analysis, several reference atlases have been made available for comparative and functional investigations of the $\mathrm{AL}$ of different insect species (Galizia et al., 1999a; Laissue et al., 1999; Berg et al., 2002; Greiner et al., 2004). The well-described anatomy of the honeybee AL and the highly invariant chemotopic representation of odors in the AL open up the possibility of investigating the equipment of ORNs of single olfactory sensilla by analyzing the glomerular pattern of stained ORNs in the AL. This approach was used in the present study.

In Hymenoptera, the olfactory sensilla house many ORNs (Dumpert, 1972; Stepper et al., 1983; Butterfield and Anderson, 1994; Ochieng et al., 2000; Isidoro et al., 2001). In honeybees, 5 to 35 ORNs are associated with the sensilla placodea (poreplate sensilla) (Schneider and Steinbrecht, 1968), and each ORN projects to the AL via one of four sensory tracts (T1-T4; Schneider and Steinbrecht, 1968; Pareto, 1972; Suzuki, 1975; Mobbs, 1982). In ants and wasps the olfactory sensilla have similar numbers of ORNs (Stepper et al., 1983; Martini and Schmidt, 1984). In some exceptional cases, e.g., some parasitic wasps (Platygastroidea), multiporus plate sensilla are equipped with up to 220 ORNs (Isidoro et al., 2001). The biological significance of multiple ORN sensilla is unknown. The large number of ORNs in Hymenoptera has so far prevented a physiological and morphological investigation of individual ORNs as was done for, e.g., the olfactory sensilla in Lepidoptera (Hansson et al., 1992, 1995; Berg et al., 1998, 2002).

In sensilla with multiple ORNs, all ORNs of one sensillum share the same sensillar lymph, which is characterized by a high concentration of potassium, supplied by the surrounding sheath cells. The high potassium concentration enhances the generation of a receptor potential during odor stimulation. Excitation of one ORN may well influence the sensillar lymph potential and, by doing so, may also influence the response of other ORNs to odors. Therefore, it seems possible that ORNs of one sensillum do not respond independently to odor stimulation. Indeed, there is some evidence that ORNs of single poreplate sensilla of the honeybee interact during odor stimulation (Akers and Getz, 1992; Getz and Akers, 1993, 1995).

A previous study showed that each ORN of a single poreplate sensillum terminates in one single glomerulus and that the innervated glomeruli are distributed across the AL (Brockmann and Bruckner, 1995). It remained unclear, however, whether different poreplate sensilla can be functionally classified according to their associated ORN types. Morphologically indistinguishable olfactory sensilla are functionally classified in Drosophila and in different moth species (Steinbrecht, 1970; Kaissling and Kasang, 1978; Meng et al., 1989; Steiner and Keil, 1995; Vosshall et al., 1999). In contrast to, e.g., the honeybee, these species have only a few (one to four) ORNs associated with one olfactory sensillum.

In the present study we investigated the glomerular pattern in the honeybee $\mathrm{AL}$ formed by the terminations of multiple ORNs housed in a single poreplate sensillum. From the different glomerular patterns found, we draw conclusions about how poreplate sensilla are equipped with different ORN types. Interindividual variance of the $\mathrm{AL}$ anatomy of different specimens often prevents the identification and comparison of individual glomeruli. Therefore, we employed an alternative method for comparison of our neuroanatomical data besides visual inspection of the AL anatomy using the two published atlases for the honeybee AL (Flanagan and Mercer, 1989; Galizia et al., 1999a).

\section{MATERIALS AND METHODS Staining procedure}

Honeybee workers (Apis mellifera carnica) were used from colonies reared at the bee station of the University of Würzburg. Workers were collected at the entrance of the hive, cooled until they became motionless, and placed into plastic holders. The head was fixed in plastic holders, and one antenna was glued horizontally onto the holder with water-soluble white-out fluid (Tipp-Ex, BIC, Clichy, France). With the help of a micromanipulator (M3301, Narishige, Tokiyo, Japan), a sharpened tungsten electrode was used to perforate a single poreplate sensillum on the flagellum between segments four and eight. The electrode was inserted into the sensillum under visual control $(70 \times)$ by using a photomicroscope (M400, Wild, Heerbrugg, Germany). Subsequently, dextran-biotin (D-7135, Molecular Probes, Eugene, OR) dissolved in aqua dest. was applied to the sensillum. Two application methods were used: 1) a glass electrode, filled with the labeling solution was inserted into the opened poreplate sensillum; or 2) a droplet of the labeling solution was applied onto the antennal segment with the opened sensillum.

The bees were kept at room temperature in a moistened chamber, and, after 24 hours, the brains were dissected in bee-ringer solution $(37.0 \mathrm{mM} \mathrm{NaCl}, 2.7 \mathrm{mM} \mathrm{KCl}, 8.0 \mathrm{mM}$ $\mathrm{Na}_{2} \mathrm{HPO}_{4}, 1.4 \mathrm{mM} \mathrm{KH} \mathrm{PO}_{4}, \mathrm{pH}$ 7.2). After dissection, brains were immediately transferred to cold $4 \%$ formaldehyde in phosphate-buffered saline (0.1 M PBS, pH 7.2) and stored for 1 hour at room temperature. Brains were then rinsed in $0.1 \mathrm{M}$ PBS $(3 \times 10$ minutes $)$, incubated in Alexa 568- or Alexa 488-conjugated streptavidin (S-11223 and S-11226, Molecular Probes) in 0.1 M PBS with $0.2 \%$ Triton X (1:125) for 48 hours, and rinsed in $0.1 \mathrm{M} \mathrm{PBS}(3 \times$ 10 minutes). Then the brains were dehydrated in an ascending series of ethanol $(50,70,80,90$, and $95 \%$ and $3 \times$ $100 \%, 10$ minutes each) and finally transferred to methylsalicylic acid (M-2047, Sigma-Aldrich, Steinheim, Germany). The brains were examined with a laser-scanning confocal microscope (Leica TCS SP, Leica Microsystems, Wetzlar, Germany).

\section{Reconstruction of glomeruli}

For reconstruction of glomeruli, the 3D software AMIRA 3.1 (Mercury Computer Systems, Berlin, Germany) was used. First, the confocal image stacks of the ALs were inspected for stained ORNs. Glomeruli that showed an innervation of ORNs were selected for reconstruction. All 
glomeruli showing an innervation with stained ORNs were labeled manually in all three planes ( $\mathrm{xy}, \mathrm{xz}, \mathrm{yz}$ ). Subsequently, the whole glomerulus was reconstructed based on the glomerulus shape in the three major axes by using the "wrap" function of AMIRA 3.1. In order to transform the ALs of different individuals (see below), landmarks were selected and labeled manually. The antennal nerve (AN), the sensory tract $\mathrm{T} 1$, and one readily identifiable glomerulus were chosen as landmarks. The landmark glomerulus was T1 (17) and A17 according to the atlases of Flanagan and Mercer (1989) and Galizia et al. (1999a). The patterns of innervated glomeruli of the ALs were visualized with sphere models by using AMIRAmol 3.1 (Mercury Computer Systems). This approach was used to evaluate by visual inspection whether repeated patterns occurred.

\section{Transformation and the VOI method}

To compare the location of innervated glomeruli in the $\mathrm{AL}$ of different individuals, all glomeruli of one $\mathrm{AL}$ were reconstructed, and the reconstructed $\mathrm{AL}$ was used as a reference AL (template AL). Eleven of the 13 ALs with stained ORNs were transformed to this template AL. The $\mathrm{AN}$, tract $\mathrm{T} 1$, and glomerulus A17 were used as landmarks. For the transformation, only the position and orientation of the specimen was changed; no scaling or deformation was used (rigid transformation). Two ALs could not be transformed because the landmarks of the AL with stained ORNs and the template AL could not be matched satisfactorily. The reconstructed glomeruli of each AL with stained ORNs were compared with the glomeruli of the template AL. The overlapping volumes of glomeruli were calculated by using the volume of interest (VOI) function of the module TissueStatistics in AMIRA 3.1. Each glomerulus with a stained ORN was mapped onto the glomerulus of the template AL with which it had the largest overlap (VOI mapping). To estimate the power of the VOI method, some glomeruli with stained ORNs were described with additional landmarks (e.g., adjacent glomeruli, sensory tracts, or individually identifiable glomeruli). Nine pairs were selected, and the glomeruli of each pair were found to be mapped onto the same glomerulus of the template AL. All possible landmarks were used to decide whether the paired glomeruli are indeed corresponding glomeruli (feature mapping). The glomeruli analyzed by feature mapping were also identified by using the atlases available for the honeybee AL.

\section{Assignment of glomeruli to sensory tracts}

The glomeruli with stained ORNs were assigned to one of the sensory tracts by a similar mapping technique as described above. Because no counterstaining could be used simultaneously for visualization of glomeruli, stained ORNs, and tract structures, the sensory tracts could not be recognized up to the glomeruli where the stained ORNs terminate. Thus, the four glomerular subpopulations, corresponding to the sensory tracts $\mathrm{T} 1-\mathrm{T} 4$, could not be distinguished. Therefore, an additional preparation with a mass staining of most ORNs was prepared. To label the sensory tracts, the flagellum was cut, and micro-ruby (D-7162, Molecular Probes) was applied to the opened antenna for 6 hours. The brain was then dissected and treated like all other specimens. The four sensory tracts and all glomeruli of this reference AL (tract AL) were reconstructed. Of all 163 glomeruli, 162 could be unambiguously assigned to one of the four sensory tracts. Only one glomerulus was difficult to assign according to the atlas of Galizia et al. (1999a). This glomerulus was most probably glomerulus D06, and we finally assigned it to T4. Most of the glomeruli were found in subpopulations T1 (72) and T3 (77); subpopulations T2 (8) and T4 (6) had only a few glomeruli.

In the next step the tract $\mathrm{AL}$ with the labeled tracts was transformed to the template AL described above, and all glomeruli of the template AL were assigned to one of the four sensory tracts by using the VOI method for glomeruli. The resulting tract assignment for the glomeruli of the template AL was then used to assign the glomeruli of the specimens with stained ORNs to the different tracts.

\section{Image editing}

The confocal images were sampled at a resolution of $1,024 \times 1,024$. For image editing we used AMIRA 3.1, Adobe Photoshop 7.0, Adobe Illustrator 10.03, and Corel Photo-Paint 11. For the image stacks shown in Figure $1 \mathrm{~A}-\mathrm{D}$, a deconvolution was calculated in AMIRA 3.1. For the enlarged sections of the AL shown in Figure $1 \mathrm{~B}$ and $\mathrm{D}$, first the resolution was increased $(4 \times)$ in Adobe Photoshop 7.0 and afterward a smoothing filter was applied in Corel Photo-Paint 11. For the reconstructed AL shown in Figure 3 , the labeled fields were down-sampled $(4 \times 4 \times 1)$ and smoothed in AMIRA 3.1. No changes in contrast were made in any of the figures shown. Numbering and lettering of all figures was done with Adobe Photoshop 7.0 and Adobe Illustrator 10.03 .

\section{Statistical analysis}

Statistical analyses were conducted with Statistica (version 6.1) software (StatSoft, Tulsa, OK). The differences in labeling treatment were tested with the t-test for independent samples. Differences in glomerular volumes were tested with a one-way ANOVA and Newman-Keuls post hoc test. Differences in relative frequencies of stained ORNs in different glomerular subpopulations were tested with Friedman's ANOVA and Wilcoxon's matched pairs as post hoc test.

\section{RESULTS}

In all, 199 ORNs from 13 individuals were stained and showed clear arborization in glomeruli of the AL. The background fluorescence was sufficient to distinguish glomeruli irrespective of whether sensory processes were stained or not (Fig. 1A-D). The AN, the sensory tract $\mathrm{T} 1$, and the soma clusters could also be discriminated. The stained ORNs could be traced in many cases (ORNs leaving $\mathrm{T} 1$ in particular) from the sensory tracts to the branching in one single glomerulus (Fig. 1F). We never observed an innervation of one glomerulus by two or more ORNs. In all cases, only a single axon entered the glomerulus and branched there. The axons of the stained ORNs branched in the cortical layers of the glomeruli, with the exception of the glomeruli innervated by the sensory tract T4. ORN axons projecting via T4 innervated the entire glomerulus (data not shown).

\section{Number of glomeruli innervated by multiple ORNs of poreplate sensilla}

We found between 7 and 23 glomeruli with stained ORNs in the 13 individuals investigated (Table 1). The two 

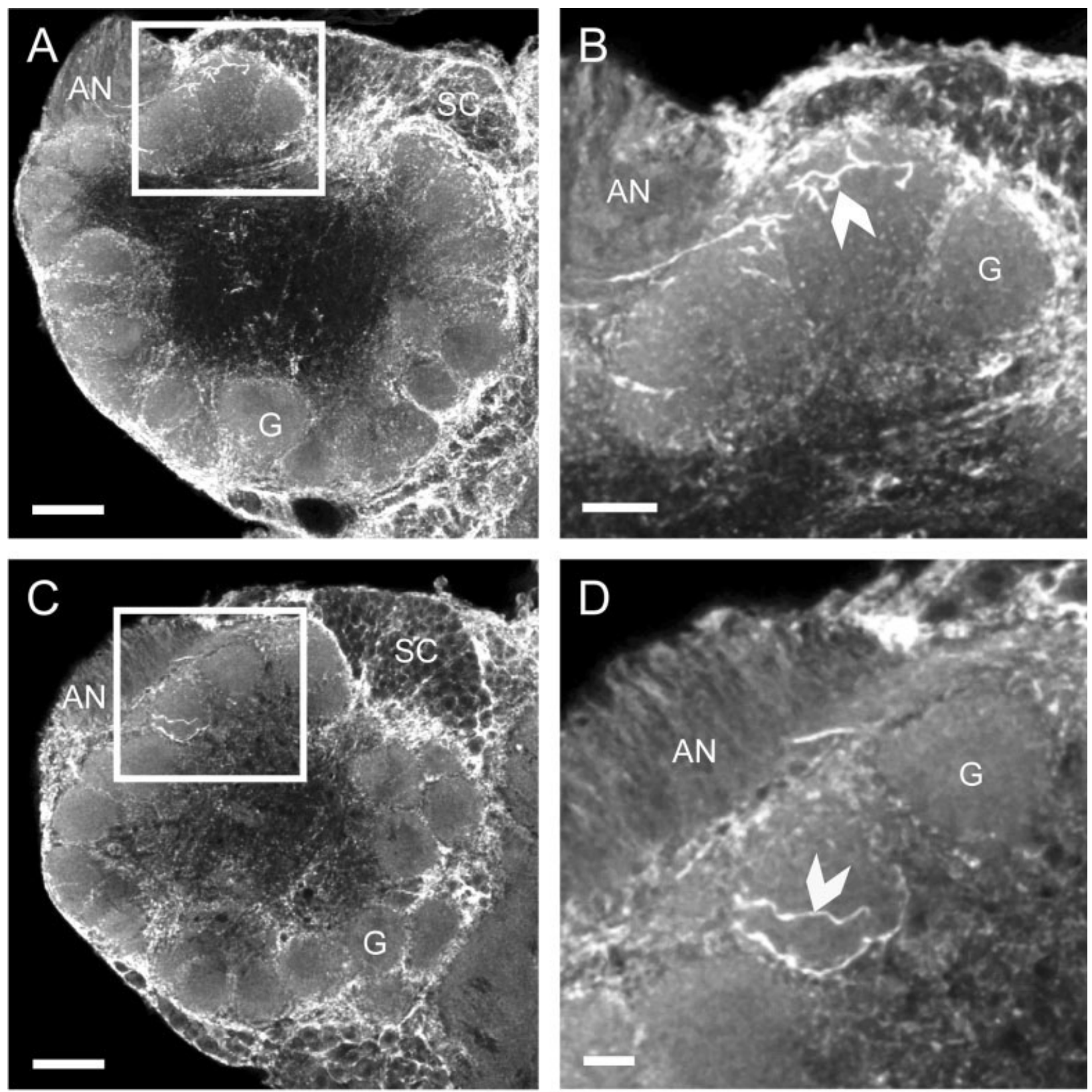

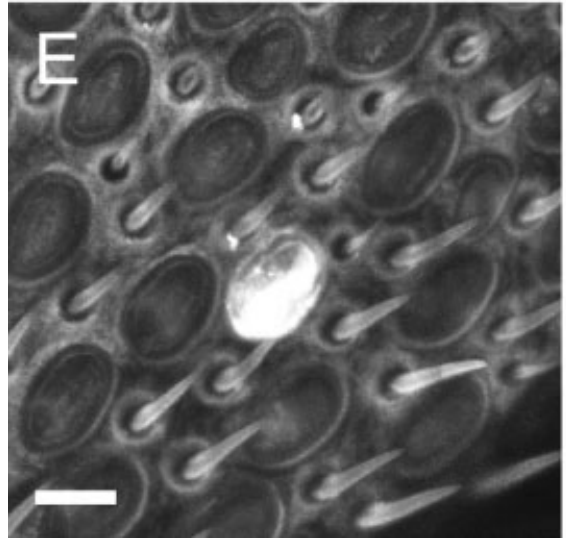

Fig. 1. Example of stained ORNs of single poreplate sensilla arborizing in glomeruli of the antennal lobe. Laser-scanning confocal micrographs of the antennal lobe of two different specimens, showing the glomeruli $(\mathbf{A}, \mathbf{C})$ and the arborization of single ORNs in glomeruli in the enlargement (B and $\mathbf{D}$, arrowheads). E: Selective staining of a single poreplate sensillum results in a bright fluorescence of the perforated sensillum, viewed with the laser-scanning microscope.
F: 3D reconstruction of three ORN axons, leaving the sensory tract and terminating in the cortical layers of three different glomeruli. The reconstruction is based on manual segmentation of all optical slices (1 $\mu \mathrm{m})$ of the confocal image stack. AN, antennal nerve; SC, soma cluster; G, glomeruli; T1, sensory tract. Scale bar $=50 \mu \mathrm{m}$ in A,C,F; $20 \mu \mathrm{m}$ in $\mathrm{B}, \mathrm{D} ; 10 \mu \mathrm{m}$ in $\mathrm{E}$. 
TABLE 1. Distribution of Stained ORNs According to the Four Sensory Tracts Innervating the ALs of 13 Different Honeybee workers ${ }^{1}$

\begin{tabular}{|c|c|c|c|c|c|c|c|}
\hline Specimen & Method & $\mathrm{T} 1$ & $\mathrm{~T} 2$ & T3 & $\mathrm{T} 4$ & $\mathrm{NN}$ & Total \\
\hline 1 & D & $5(6.9)$ & $0(0)$ & $7(9.7)$ & $0(0)$ & & 12 \\
\hline 2 & D & $7(9.6)$ & $0(0)$ & $6(8.3)$ & $0(0)$ & & 13 \\
\hline 3 & D & $5(6.9)$ & $0(0)$ & $8(11.1)$ & $0(0)$ & 2 & 15 \\
\hline 4 & D & $1(1.4)$ & $0(0)$ & $7(9.7)$ & $0(0)$ & & 8 \\
\hline 5 & D & $2(2.7)$ & $0(0)$ & $13(18.1)$ & $0(0)$ & 1 & 16 \\
\hline 6 & D & $7(9.6)$ & $0(0)$ & 9 (12.5) & $0(0)$ & 2 & 18 \\
\hline 7 & D & & & & & 19 & 19 \\
\hline 8 & GE & $15(20.6)$ & $1(12.5)$ & $1(1.4)$ & $2(18.2)$ & 2 & 21 \\
\hline 9 & GE & $4(5.5)$ & $1(12.5)$ & $2(2.8)$ & $0(0)$ & & 7 \\
\hline 10 & GE & $8(11.0)$ & $0(0)$ & $2(2.8)$ & $2(18.2)$ & & 12 \\
\hline 11 & GE & 10 (13.7) & $0(0)$ & $1(1.4)$ & $0(0)$ & 2 & 13 \\
\hline 12 & $\mathrm{GE}$ & $12(16.4)$ & $1(12.5)$ & $5(6.9)$ & $1(9.1)$ & 3 & 22 \\
\hline 13 & GE & & & & & 23 & 23 \\
\hline Total & & 76 & 3 & 61 & 5 & 54 & 199 \\
\hline
\end{tabular}

${ }^{1} \mathrm{D}$, application of the staining solution with a droplet; GE, application of the staining solution with a glass electrode; T1-T4, sensory tracts; NN, no assignment to a glomerular subpopulation or not mapped to the template AL; number in brackets, relative ular subpopulation or not mapped to the template AL; number in brackets, relative
frequency of stained ORNs (glomeruli) related to the total number of glomeruli belonging to each tract in the template $\mathrm{AL}(\%)$.

different methods used to apply the labeling solution had no obvious influence on the mean number of stained ORNs which was 14.43 with the droplet method $(\mathrm{n}=7)$ and 16.33 with glass electrodes $(n=6$; t-test for independent samples; $P=0.21$ ).

We perforated a single poreplate sensillum under optical control with a tungsten electrode. However, we had to ensure that with the method used, only one sensillum was opened and that the application of the labeling solution was restricted to the ORNs of this sensillum. We investigated the antennae of some individuals with successfully stained ORNs. The antennae were treated like the dissected brains and viewed with the confocal microscope. In two of seven cases, we were able to localize the perforated poreplate sensillum on the antennal surface by its bright fluorescence (Fig. 1E). All sensilla in the surrounding of the perforated sensillum were dark and still intact. In the remaining five cases no sensillum with bright fluorescence could be detected.

\section{Glomerular pattern of stained ORNs}

To compare the glomerular patterns of ORN projections from single poreplate sensilla, we visualized the glomeruli with sphere models. We never found two identical or highly similar glomerular patterns. As an example, 3 of 11 glomerular patterns are shown in Figure 2. Some glomerular patterns have similar features, e.g., clusters or rows of glomeruli. In three specimens the innervated glomeruli are separated from each other by noninnervated glomeruli (Fig. 2A). In three specimens, we found that most of the innervated glomeruli were in a row (Fig. 2B). In four other specimens, we found most of the innervated glomeruli in a cluster (Fig. 2C). Although some features of the glomerular patterns were found repeatedly, the diversity of the patterns prevents an unambiguous classification. Such diversity shows that there is no simple organization principle by which specific ORN types are assigned to poreplate sensilla.

\section{Overlap of glomerular patterns}

To detect a possible overlap between the glomerular patterns formed by ORNs of different poreplate sensilla, we analyzed the glomerular patterns using VOI mapping. A map was created, showing the distribution of innervated glomeruli of all specimens superimposed on the glomeruli of the template AL (Fig. 3A). We found that the innervated glomeruli are distributed across the entire AL, and the anterior side of the AL seemed to have more innervated glomeruli (see next paragraph). Some glomeruli of the template $\mathrm{AL}$ had a major overlap with, e.g., one stained glomerulus in up to six specimens (Fig. 3, red glomeruli, and Table 2).The alignment of the AL with stained glomeruli to the template AL was solely based on three landmarks (AN, T1, and the glomerulus A17). All other morphological features were not considered in the VOI mapping.

To evaluate the accuracy of the VOI mapping, we compared the results with a glomerulus identification in which we used several characteristic landmarks in the surrounding of the glomeruli of interest (feature mapping). Figure 3B shows an example of one pair of glomeruli ( $\mathrm{X}$ and $\mathrm{Y}$ ) in which, in addition to the $\mathrm{AN}$ and $\mathrm{T} 1$, one characteristic glomerulus $(\mathrm{G})$ and the lateral soma cluster (l-SC) were also reconstructed and used for identification. According to the morphological features used, the glomeruli of interest (X and Y) are corresponding glomeruli as they also are by VOI mapping. Feature mapping revealed corresponding glomeruli for seven of nine pairs. In the remaining two pairs the glomeruli could not be identified as corresponding glomeruli. In some cases individual glomeruli could be identified individually according to the published honeybee AL atlases (Flanagan and Mercer, 1989; Galizia et al., 1999a). This was possible for, e.g., glomerulus $\mathrm{G}$ in Figure 3B, which corresponds to T3 (2) and $\mathrm{C} 02$, respectively, and glomeruli $\mathrm{X}$ and $\mathrm{Y}$, which correspond to $\mathrm{T} 1$ (4) and A04, respectively (Table 2).

\section{Innervation pathway of ORNs into the $\mathrm{AL}$}

Each glomerulus of the AL belongs to one subpopulation of glomeruli, defined by its innervation of ORNs via one of the four sensory tracts (T1-T4). We analyzed the distribution of ORNs from one poreplate sensillum according to their innervation pathway via one of the four sensory tracts. The mapping of ORNs to tracts was done with the $\mathrm{AL}$ containing mass-labeled tracts and VOI mapping to assign all glomeruli of the template AL to one of the four sensory tracts. Most of the glomeruli were assigned to sensory tracts $\mathrm{T} 1$ and $\mathrm{T} 3$ (73 and 72 glomeruli, respectively), 8 glomeruli were assigned to T2, and 11 glomeruli were assigned to $\mathrm{T} 4$. The average volume of the $\mathrm{T} 4$ glomeruli was larger than the average volume of glomeruli of all three other subpopulations (one-way ANOVA; $\mathrm{F}_{3,159}=$ 36.35; $P<0.01$ and Newman-Keuls post hoc test). In the template AL we did not find any difference in volume between the glomeruli of the four (assigned) subpopulations (one-way ANOVA; $\mathrm{F}_{3,160}=0.51 ; P=0.68$ ).

In all specimens in which mapping to the template $\mathrm{AL}$ was possible $(\mathrm{n}=11)$, we found innervated glomeruli belonging to more than one subpopulation (Table 1). The sensory tracts T1 and T3 were the most frequently found innervation pathways ( $\mathrm{T} 1=76 \mathrm{ORNs}$ and $\mathrm{T} 3=61 \mathrm{ORNs}$ ). Only two specimens showed glomerular innervations via all four sensory tracts. We compared the relative frequency of stained glomeruli for the four subpopulations and found differences (Friedman's ANOVA; $\mathrm{CHI}^{2}=12.15$; $\mathrm{FG}=3 ; P<0.01)$. The post hoc test revealed that the glomerular subpopulation of $\mathrm{T} 1$ was more often inner- 

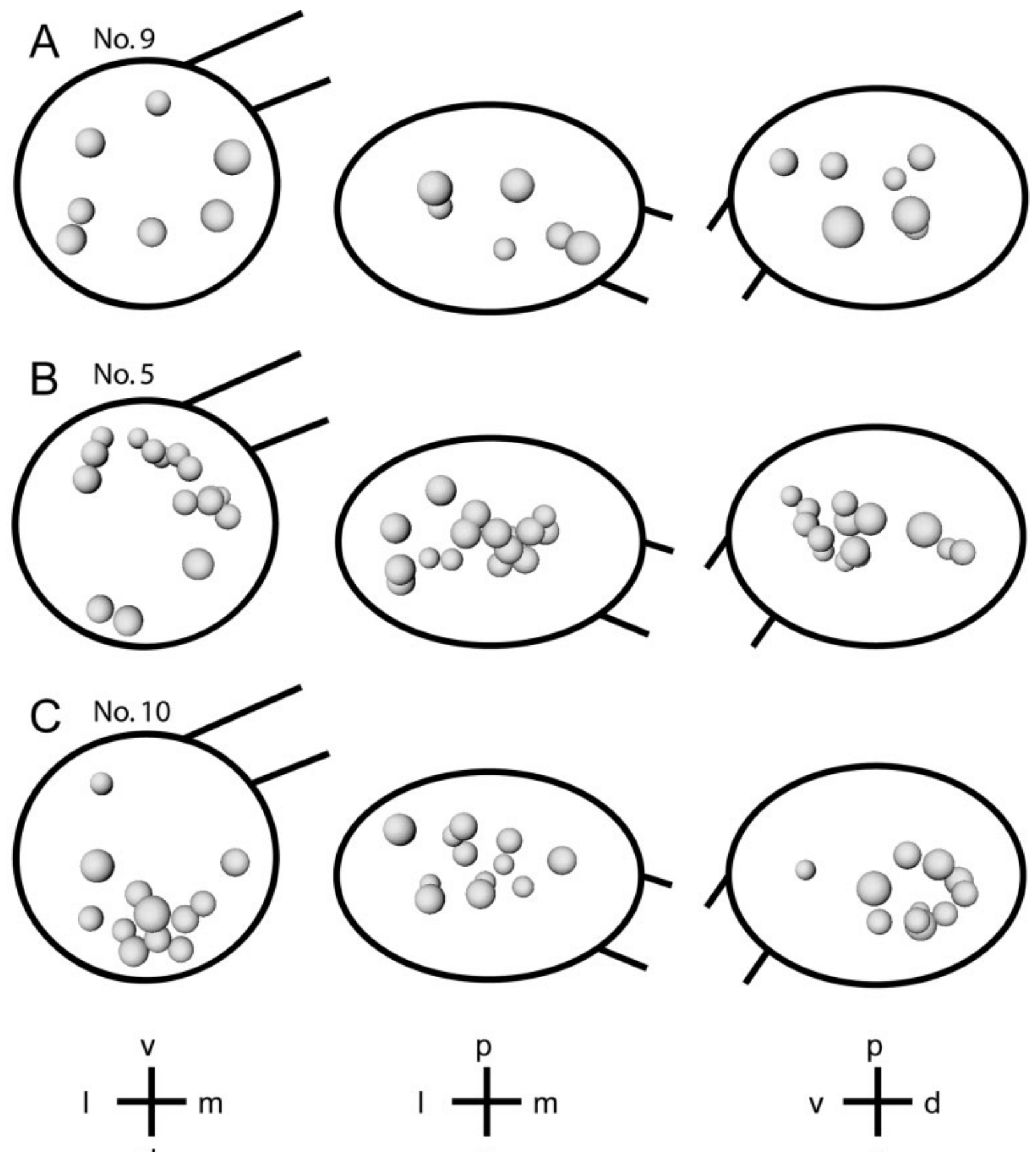

Fig. 2. Sphere models of the glomerular patterns in the AL, defined by the innervation of ORNs of three different poreplate sensilla (A, specimen 9; B, specimen 5; and $\mathbf{C}$, specimen 10; see Table 1) from three different points of view. Some specimens had glomerular patterns with separated glomeruli as in A; in other specimens the glo-

meruli were in a row as in B or the glomeruli were clustered as in $\mathrm{C}$. The profiles of the AL and the antennal nerve are schematically outlined. v, ventral; m, median; d, dorsal; l, lateral. Scale bar $=100$ $\mu \mathrm{m}$ (applies to all).

vated than the glomerular subpopulations T2 and T4 (Wilcoxon matched pairs test; $\mathrm{n}=11 ; \mathrm{Z}=2.40$ and $\mathrm{Z}=2.31$; $P<0.05$ for both pairs). For all other pairs we found no difference.

\section{DISCUSSION}

We investigated the glomerular projections of multiple ORNs housed in poreplate sensilla. Our results allow us to 

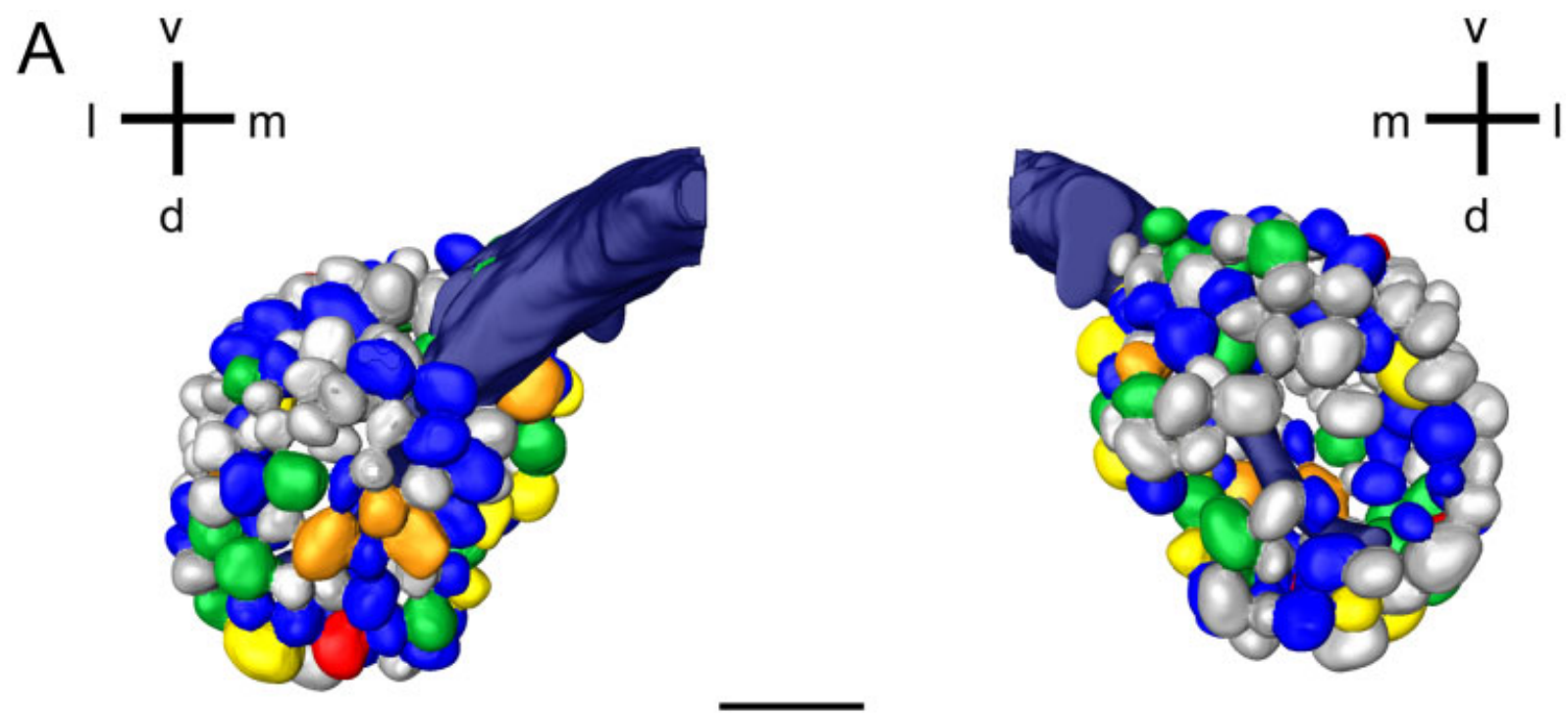

B
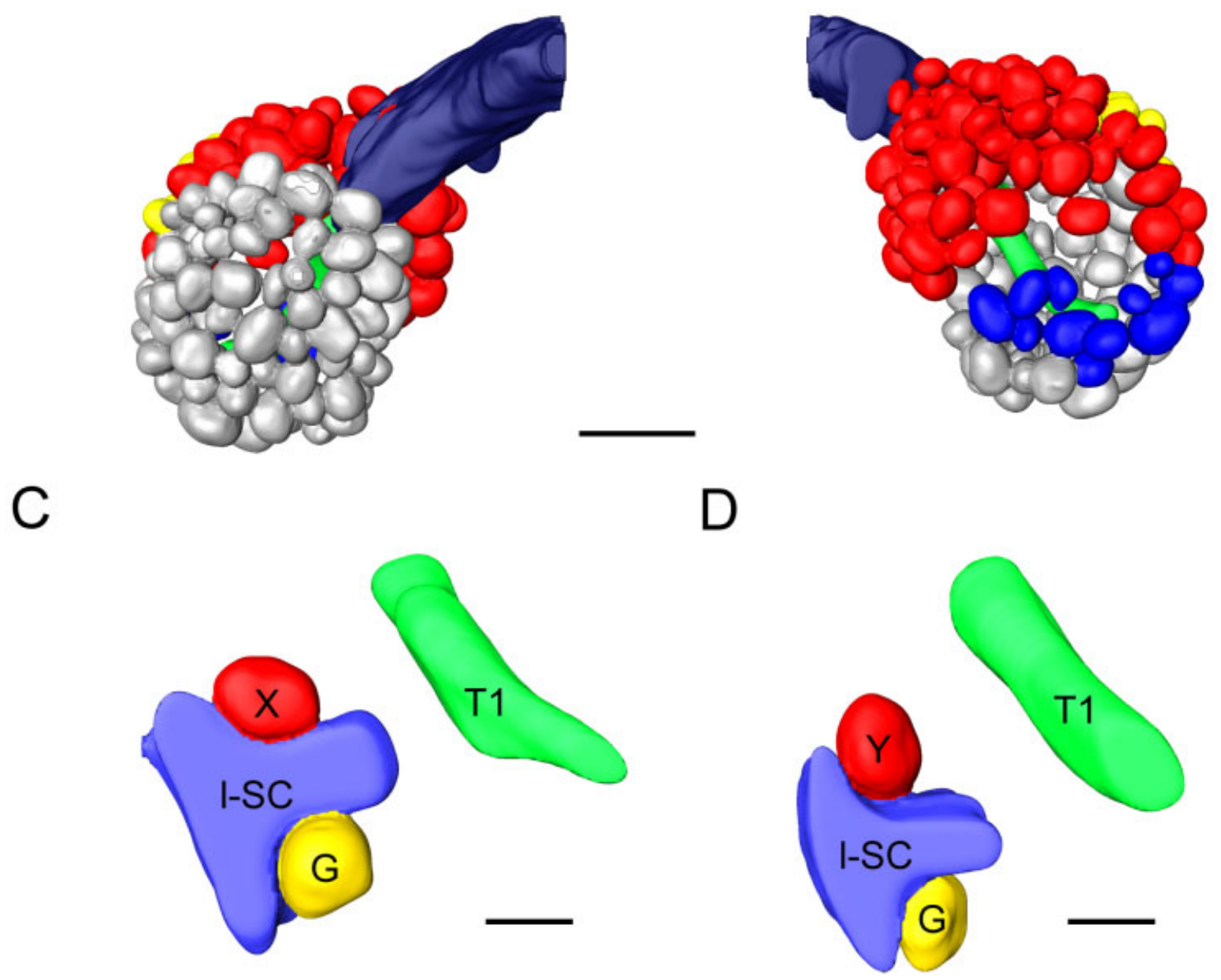

Fig. 3. Glomerular patterns of arborizing ORNs of different specimens (A) and of different sensory tracts (B) mapped onto a reconstructed reference AL (template AL). A: ORNs arborize in different glomeruli, distributed over the entire AL (blue, red). The anterior part of the AL with the glomeruli of the T1 subpopulation (left) shows more innervated glomeruli than the posterior part (right). The glomerular patterns are overlapping. Single glomeruli of several investigated specimens were mapped onto the same glomerulus of the template AL (red $=5-6 ;$ orange $=4 ;$ yellow $=3$ green $=2 ;$ blue $=1$; grey $=0 ;$ dark blue $=$ antennal nerve and sensory tract T1). B: All glomeruli of the template AL were assigned to one of the four sensory tracts by VOI

mapping of the tract AL. The glomeruli of the subpopulation T1 are located anterior (left: gray $=\mathrm{T} 1$ ), the subpopulations T3 and T4 are located posterior (right: red $=\mathrm{T} 3$, blue $=\mathrm{T} 4$ ), and the subpopulation T2 is located lateral (yellow = T2). C,D: Example of one selected pair of glomeruli ( $\mathrm{X}$ in $\mathrm{C}$ and $\mathrm{Y}$ in $\mathrm{D}$ ) of which both were mapped onto the same glomerulus of the template AL by VOI mapping. X and Y were also compared by visual inspection (feature mapping) and identified as corresponding glomeruli. l-SC, lateral soma cluster; G, glomeruli; $\mathrm{T} 1$, sensory tract; $\mathrm{X}$ and $\mathrm{Y}$, glomeruli of interest; $\mathrm{v}$, ventral; $\mathrm{m}$, median; d, dorsal; 1, lateral. Scale bar $=100 \mu \mathrm{m}$ in A,B; $20 \mu \mathrm{m}$ in C,D. 
TABLE 2. Distribution of Overlapping Innervations of the Same Glomerulus According to the Four Sensory Tracts ${ }^{1}$

\begin{tabular}{ccccc}
\hline Overlap & T1 & T2 & T3 & T4 \\
\hline 0 & 31 & 6 & 38 & 5 \\
1 & 24 & 2 & 18 & 4 \\
2 & $8(\mathrm{~A} 17, \mathrm{~A} 04)$ & 0 & $10(\mathrm{C} 02)$ & 1 \\
3 & 4 & 0 & 3 & 1 \\
4 & 4 & 0 & 2 & 0 \\
5 & 1 & 0 & $1(\mathrm{C} 62)$ & 0 \\
6 & 1 & 0 & 0 & 0
\end{tabular}

${ }^{1}$ Overlap, number of specimens in which an innervation of the same glomerulus was found; T1-T4, sensory tracts; numbers in brackets, glomeruli located according to the published honeybee AL atlases (Flanagan and Mercer, 1989; Galizia et al., 1999a).

draw conclusions about the equipment of ORN types in individual poreplate sensilla. This is possible because functionally corresponding glomeruli are almost invariant in relative position to each other across individuals (Galizia et al., 1999b). We found that ORNs of single poreplate sensilla form different glomerular patterns in the AL. The equipment of poreplate sensilla with ORNs is overlapping; different poreplate sensilla share some of the many different ORN types found in one sensillum. The glomerular patterns formed by the innervation of ORNs in the $\mathrm{AL}$ were compared across individuals by using different methods. The alignment of ALs according to three landmarks and the mapping of innervated glomeruli (VOI mapping) to a reference $\mathrm{AL}$ (template $\mathrm{AL}$ ) proved to be especially useful for interindividual comparison. This method might also be useful for comparative neuroanatomical studies of other neuropils as complex as the ALs of honeybees.

\section{Selective staining of ORNs}

We stained the ORNs of single poreplate sensilla under optical control and, in addition, we were able to confirm that only single sensilla were stained with this method. Only ORNs of perforated sensilla were stained, even if a droplet for dye application was used. No receptor neurons were stained when a droplet of labeling solution was applied without perforating a sensillum. Mechanosensory neurons are stained when large quantities of labeling solution are applied to the antenna. This was never the case in our staining of receptor neurons. Thus, we are confident that our staining method was selective for single poreplate sensilla.

Staining of ORNs with dextran-biotin and Alexaconjugated streptavidin allowed laser-scanning confocal microscopy, which has the major advantage that the neuropil can be scanned and analyzed as a whole. Recently, tetramethylrhodamine dextran was used successfully for staining ORNs of single sensilla (Stranden et al., 2003). It seems that the dextran, coupled to biotin in our case, is crucial for transport to the axon terminals of the ORNs. So far, the most commonly used technique to study ORN arborization in insects has been labeling with cobalt (cobalt lysine or cobalt chlorite) and subsequent light microscopy (Hansson et al., 1992, 1995; Boeckh and Tolbert, 1993; Stocker, 1994; Brockmann and Bruckner, 1995; Berg et al., 1998, 2005). Although the quality of staining is comparable in both techniques, the data analysis of cobalt staining of ORNs is limited and thus prone to interpretation errors.

We found between 7 and $23 \mathrm{ORNs}$ in 13 different poreplate sensilla. This number corresponds well to results based on the ultrastructure of poreplate sensilla showing that 5-35 receptor neurons are associated with one sensillum (Schneider and Steinbrecht, 1968). Our method seems to label most if not all ORNs of a single poreplate sensillum. This is not necessarily the case with cobalt staining; the technique was even used to label excited neurons selectively during dye application (Hansson et al., 1992, 1995; Ochieng et al., 1995).

\section{Glomerular pattern of multiple ORNs}

The 199 ORNs investigated in this study innervate the AL uniglomerularly and in glomeruli distributed across the whole AL. This result confirms a previous study (Brockmann and Bruckner, 1995), with the exception that even with the much larger number of ORNs analyzed in our study we never found an ORN terminating in more than one glomerulus. The $3 \mathrm{D}$ reconstruction of innervated glomeruli (sphere models) allowed visual inspection of the glomerular pattern in the AL. This revealed a diversity of glomerular patterns formed by ORNs of different poreplate sensilla. We could not find a common organization of glomerular patterns nor did we find repeated patterns. The diversity we found indicates that if repeated glomerular patterns exist at all, they are rare. It rather seems that poreplate sensilla are individually equipped with different ORNs. However, if many different but repeated functional types of poreplate sensilla exist, our limited sample size of 13 specimens may not be sufficient to find the same glomerular pattern twice.

\section{Comparison of different specimens}

To compare the ORN equipment of poreplate sensilla of different specimens, we used a 3D analysis method based on a few easily detectable landmarks (VOI mapping). We then compared this approach with traditional visual inspection and mapped the glomeruli of interest according to characteristic features (landmarks) found in the AL (feature mapping). As landmarks, sensory tracts, somata clusters, and primary glomeruli (term from Rospars and Hildebrand, 1992) were used. The results were also compared with the two AL atlases available (Flanagan and Mercer, 1989; Galizia et al., 1999a). It was proposed by the authors that most of the glomeruli in the AL can be identified individually, although variations in relative position and number of glomeruli were found. The variation in, e.g., the number of glomeruli found in the AL of different workers ranges from 71 to 77 for the glomerular subpopulation T1 (Arnold et al., 1985; Flanagan and Mercer, 1989; Galizia et al., 1999a). We found it very difficult to identify most of the glomeruli individually with the help of the atlases. This was mainly because of the large number of glomeruli $(\sim 167)$ in honeybees, the variance between specimens, and the lack of unambiguous landmarks. Individual identification was possible for some glomeruli in the characteristic glomerular arrangement and close to landmarks, e.g., sensory tracts. The comparison between VOI mapping and feature mapping revealed that the glomeruli identified as corresponding glomeruli were the same in seven of nine cases. For the VOI mapping, only a few landmarks and no further classification of AL structures are necessary.

One advantage of this approach is that it can be used without identification of any other AL structures beside the (few) landmarks. Thus, the VOI mapping as used in this study is based on less interpretation of anatomical data than, e.g., the feature mapping. A similar approach 
to a comparison of the positions of axonal projections of ORNs was recently used in Anopheles gambiae (Anton and Rospars, 2004). In contrast to our study, scaling of the specimens (nonrigid transformation) was necessary due to considerable variation of brain size in Anopheles. The axonal projections of the ORNs were compared directly, whereas we compared the functional units of the $\mathrm{AL}$, the glomeruli.

Our study shows that VOI mapping is an adequate and fast method for comparing, e.g., glomerular patterns in the $\mathrm{AL}$ of the honeybee.

\section{Overlap of glomerular patterns}

VOI mapping of glomerular patterns revealed that repeatedly labeled glomeruli were found at the same spatial position in the AL. Functionally corresponding glomeruli are almost invariant in relative position to each other across individuals (Galizia et al., 1999b). A mismatch between spatial position and function of a glomerulus across individuals can be caused by interindividual variance in the AL design. Unfortunately, no quantitative data are available showing how variable the AL design actually is in different individuals of the honeybee. In addition to this variation, our data analysis might have caused a mismatch by an incorrect transformation of the specimens. The comparison of VOI mapping and feature mapping (see "Comparison of different specimens") showed that the different specimens are well aligned after rigid transformation. Our measure of overlap is based solely on the spatial position of glomeruli in the $\mathrm{AL}$, and no direct measure of the physiology of the stained ORNs is available at present. Thus, we cannot be sure that the overlapping innervated glomeruli when superimposed on the template AL are all functionally the same glomeruli. However, the large numbers of overlapping glomeruli indicate that in addition to the spatial overlap a functional overlap of ORN types also exists. One glomerulus of the template $\mathrm{AL}$ had a major overlap to one glomerulus in $55 \%(6 / 11)$ of the specimens with stained ORNs. A spatial overlap of glomerular pattern was suggested previously (Brockmann and Bruckner, 1995), and our present study shows that indeed such an overlap exists. From our data we conclude that poreplate sensilla are equipped with different ORN types but that the same ORN type can be found in different poreplate sensilla.

\section{Innervation pathways of ORNs via T1-T4}

We assigned all glomeruli of the template AL to one of the four sensory tracts innervating the AL. With 73, 8, 72, and 11 glomeruli found for sensory tracts T1, T2, T3, and $\mathrm{T} 4$, respectively, the numbers of glomeruli we found in the template $\mathrm{AL}$ correspond well to results from previous studies (Arnold et al., 1985; Flanagan and Mercer, 1989; Galizia et al., 1999a). We are confident that most of the glomeruli of the large (T1 and T3) subpopulations are assigned correctly to the sensory tracts. Primary glomeruli that could be identified individually with the help of the atlases in both ALs (tract and template AL) were compared, and no mismatching was found. The assignment of the glomeruli of subpopulation T4 is supposedly overestimated, because tract $\mathrm{AL}$ had large glomeruli in this subpopulation. In the template $\mathrm{AL}$ we did not find enlarged glomeruli in the T4 region. The difference between these two ALs probably reflects the interindividual variation, which might be, e.g., experience dependent
(Winnington et al., 1996; Sigg et al., 1997; Brown et al., $2002,2004)$. The arborization of ORNs in the glomeruli that we assigned to subpopulation T4 differed from the arborization of all other ORNs investigated. In contrast to the ORNs of sensory tracts T1-T3, the ORNs of the T4 subpopulation innervate not only the cortical layer but the entire glomerulus (Arnold et al., 1985). The innervation pattern of ORNs that we assigned to the T4 subpopulation showed that indeed their axons branch in the entire glomerulus, confirming our correct assignment.

We analyzed the distribution of ORNs of single poreplate sensilla to the four sensory tracts. Compared with the number of glomeruli for each subpopulation, ORNs innervate the AL more often via sensory tract $\mathrm{T} 1$ than via T2 and T4. Sensory tract T3 seems to be a relatively frequent pathway for ORNs; however, the difference to the other pathways (T2 and T4) was not statistically significant. Our supposed overestimation of the number of glomeruli of subpopulation T4 might have added to the difference found, but the difference between the innervation via sensory tracts $\mathrm{T} 1$ and $\mathrm{T} 2$ should be unaffected by this possible error.

Our results show that ORNs of single poreplate sensilla innervate different glomeruli via more than one sensory tract. All sensory tracts in the AL (T1-T4) are possible pathways for ORNs of poreplate sensilla; however, sensory tracts $\mathrm{T} 1$ and possibly T3 are more common innervation pathways. The disproportional distributions of ORNs to the four glomerular subpopulations indicate that the equipment of poreplate sensilla with ORN types is not arbitrary. Some ORN types were found with a lower frequency (T2 and T4) than others (e.g., T1). It remains to be investigated whether the ORN types found with a lower frequency are also less abundant in the antenna, or, e.g., whether other types of sensilla, beside the poreplate sensilla, house these ORN types.

\section{Possible functional significance of multiple ORNs}

The olfactory sensilla in Hymenoptera are equipped with multiple ORNs. The surface area on the antenna occupied by one poreplate sensillum (diameter $\sim 9 \mu \mathrm{m}$ ) is slightly larger than, e.g., the area occupied by a basiconic sensillum (diameter $\sim 8 \mu \mathrm{m}$ ) (Esslen and Kaissling, 1976). Poreplate sensilla are also found in scarab beetles; in Popillia japonica, for example, the poreplate sensilla are equipped with only two ORNs and range from 9 to $19 \mu \mathrm{m}$ in diameter (Agren, 1985). In comparison, the large basiconic sensilla in Drosophila are equipped with two or four ORNs and are about $7 \mu \mathrm{m}$ in diameter at the cup-shaped base of the sensillum (Shanbhag et al., 1999). We assume that the olfactory sense organs of both Drosophila and the honeybee are compound noses (Hekmat-Scafe et al., 1998) with a maximum extent of miniaturization. The sensilla density on the funiculus of Drosophila and on the flagellum of the honeybee differs. Calculated from literature data (Shanbhag et al., 1999), the density of sensilla of all types on the funiculus of Drosophila is about 3,000 sensilla/ $/ \mathrm{mm}^{2}$ compared with only 700 poreplates $/ \mathrm{mm}^{2}$ on the flagellum of the honeybee (Esslen and Kaissling, 1976). The lower number of sensilla in honeybees seems to be overcompensated by the multiple ORNs of poreplate sensilla. With a $\sim 10$ times larger surface area of the flagellum, honeybees have $\sim 50$ times more ORNs than Drosophila on its funiculus. Thus, an increase in ORN 
number might be one possible reason for the evolution of multiple ORN sensilla. A larger number of ORNs (with the same specificity) increases the sensitivity of the compound nose for two reasons.

The probability that an odor molecule is being received increases with increasing number of ORNs, and the signal to noise ratio is improved by more ORNs. The fact that poreplate sensilla are equipped with different types of ORNs, as we described in this study, does not compromise these advantages as long as ORNs are responding to odors independently of each other. Indeed, this might not be the case. Interactions between ORNs have been shown for moths and honeybees. O'Connell and colleagues (1986) examined the responses of pheromone-sensitive ORNs in moths and found mixture interactions at the level of receptor neurons. Furthermore, synergistic effects between ORNs for sex pheromone compounds and ORNs for host plant volatiles have been verified (Ochieng et al., 2002). In the honeybee, the first evidence for interactions between ORNs was discussed by Vareschi (1971). Later, synergistic and inhibitory effects between subsets of ORNs were described by extracellular recordings of ORNs of single poreplate sensilla (Akers and Getz, 1992; Getz and Akers, 1993, 1995).

The interactions between ORNs are not restricted to multicomponent odors. ORNs of poreplate sensilla are broadly tuned to odors (Lacher, 1964; Vareschi, 1971). Because ORNs with different odor specificities are associated with one poreplate sensillum, several of these ORNs respond to a single-component odor and might interact with each other (Getz and Akers, 1993). Odor discrimination is not necessarily impaired by interaction of ORNs. Interactions between ORNs are only disadvantageous if the interacting ORNs have the same or very similar specificity and sensitivity, a situation so far not found in any of the sensilla investigated (Vermeulen and Rospars, 2004). Rather than degrading olfactory perception, interaction of ORNs might actually enhance olfactory perception of, e.g., multicomponent odors by assigning an assembly of excited ORNs that is distinct from the assembly of ORNs excited by its single-component odors. The olfactory system shows such neuronal processing in the AL where the representation of odors appeared to be more confined at the level of the projection neurons than at the level of the receptor neurons (Sachse and Galizia, 2002, 2003). It remains to be investigated whether functionally similar processing of odor information already takes place at the level of single sensilla.

\section{ACKNOWLEDGMENTS}

We are deeply grateful to S. Kirschner for staining and analysis of the antennal lobe tract. We thank M. Obermayer for technical assistance. We also thank D. Ahrens and H. Demmel of the bee station (University of Würzburg) for the supply of bees.

\section{LITERATURE CITED}

Agren L. 1985. Architecture of a lamellicorn flagellum (Phyllopertha horticola, Scarabaeidae, Coleoptera, Insecta). J Morphol 186:85--94.

Akers RP, Getz WM. 1992. A test of identified response classes among olfactory receptor neurons in the honeybee worker. Chem Senses 17: 191-209.

Anton S, Rospars JP. 2004. Quantitative analysis of olfactory receptor neuron projections in the antennal lobe of the malaria mosquito, Anopheles gambiae. J Comp Neurol 475:315-326.

Arnold G, Masson C, Budharugsa S. 1985. Comparative study of the antennal lobes and their afferent pathways in the worker bee and the drone (Apis mellifera). Cell Tissue Res 242:593-605.

Berg BG, Almaas TJ, Bjaalie JG, Mustaparta H. 1998. The macroglomerular complex of the antennal lobe in the tobacco budworm moth $\mathrm{He}$ liothis virescens: specified subdivision in four compartments according to information about biologically significant compounds. J Comp Physiol A 183:669-682.

Berg BG, Galizia CG, Brandt R, Mustaparta H. 2002. Digital atlases of the antennal lobe in two species of tobacco budworm moths, the oriental Helicoverpa assulta (male) and the American Heliothis virescens (male and female). J Comp Neurol 446:123-134.

Berg BG, Almaas TJ, Bjaalie JG, Mustaparta H. 2005. Projections of male-specific receptor neurons in the antennal lobe of the oriental tobacco budworm moth, Helicoverpa assulta: a unique glomerular organization among related species. J. Comp. Neurol. 486:209-220.

Boeckh J, Tolbert LP. 1993. Synaptic organization and development of the antennal lobe in insects. Microsc Res Techn 24:260-280.

Brockmann A, Bruckner D. 1995. Projection pattern of poreplate sensory neurons in honey-bee worker, Apis mellifera L (Hymenoptera, Apidae). Int J Insect Morphol Embryol 24:405-411.

Brown SM, Napper RM, Thompson CM, Mercer AR. 2002. Stereological analysis reveals striking differences in the structural plasticity of two readily identifiable glomeruli in the antennal lobes of the adult worker honeybee. J Neurosci 22:8514-8522.

Brown SM, Napper RM, Mercer AR. 2004. Foraging experience, glomerulus volume, and synapse number: a stereological study of the honey bee antennal lobe. J Neurobiol 60:40-50.

Butterfield A, Anderson M. 1994. Morphology and ultrastructure of antennal sensilla of the parasitoid, Trybliographa rapae (Westw) (Hymenoptera, Cynipidae). Int J Insect Morphol Embryol 23:11-20.

Dumpert K. 1972. Receptors for alarm substances on antenna of Lasius fuliginosus (Latr) (Hymenoptera, Formicidae). Z Vergl Physiol 76:403.

Esslen J, Kaissling KE. 1976. Number and distribution of sensilla on antennal flagellum of honeybee (Apis mellifera L). Zoomorphology 83: $227-251$.

Flanagan D, Mercer AR. 1989. An atlas and 3-D reconstruction of the antennal lobes in the worker honey bee, Apis mellifera (Hymenoptera, Apidae). Int J Insect Morphol Embryol 18:145-159.

Galizia CG, McIlwrath SL, Menzel R. 1999a. A digital three-dimensional atlas of the honeybee antennal lobe based on optical sections acquired by confocal microscopy. Cell Tissue Res 295:383-394.

Galizia CG, Sachse S, Rappert A, Menzel R. 1999b. The glomerular code for odor representation is species specific in the honeybee Apis mellifera. Nat Neurosci 2:473-478.

Getz WM, Akers RP. 1993. Olfactory response characteristics and tuning structure of placodes in the honey-bee Apis mellifera L. Apidologie 24:195-217.

Getz WM, Akers RP. 1995. Partitioning nonlinearities in the response of honey-bee olfactory receptor neurons to binary odors. Biosystems 34 : 27-40.

Greiner B, Gadenne C, Anton S. 2004. Three-dimensional antennal lobe atlas of the male moth, Agrotis ipsilon: a tool to study structurefunction correlation. J Comp Neurol 475:202-210.

Hansson BS, Ljungberg H, Hallberg E, Lofstedt C. 1992. Functional specialization of olfactory glomeruli in a moth. Science 256:1313-1315.

Hansson BS, Almaas TJ, Anton S. 1995. Chemical communication in heliothine moths. 5. Antennal lobe projection patterns of pheromonedetecting olfactory receptor neurons in the male Heliothis virescens (Lepidoptera, Noctuidae). J Comp Physiol A:535-543.

Hekmat-Scafe DS, Steinbrecht RA, Carlson JR. 1998. Olfactory coding in a compound nose- coexpression of odorant-binding proteins in Drosophila. In: Olfaction and taste XII. Ann NY Acad Sci 855:311-315.

Hildebrand JG, Shepherd GM. 1997. Mechanisms of olfactory discrimination: converging evidence for common principles across phyla. Annu Rev Neurosci 20:595-631.

Isidoro N, Romani R, Bin F. 2001. Antennal multiporous sensilla: their gustatory features for host recognition in female parasitic wasps (Insecta, Hymenoptera: Platygastroidea). Microsc Res Techn 55:350-358.

Joerges J, Küttner A, Galizia GC, Menzel R. 1997. Representation of odours and odour mixtures visualized in the honeybee brain. Nature 387:285-288.

Kaissling KE, Kasang G. 1978. New pheromone of silkworm moth Bombyx 
mori-sensory pathway and behavioral effect. Naturwissenschaften 65:382-384.

Kleineidam CJ, Obermayer M, Halbich W, Rössler W. 2005. A macroglomerulus in the antennal lobe of leaf-cutting ant workers and its possible functional significance. Chem Senses 30:383-392.

Lacher V. 1964. Elektrophysiologische Untersuchungen an einzelnen Rezeptoren für den Geruch, Kohlendioxid, Luftfeuchtigkeit und Temperatur auf den Antennen der Arbeitsbiene und der Drohne (Apis mellifica L.). Z Vergl Physiol 48:587-623.

Laissue PP, Reiter C, Hiesinger PR, Halter S, Fischbach KF, Stocker RF. 1999. Three-dimensional reconstruction of the antennal lobe in Drosophila melanogaster. J Comp Neurol 405:543-552.

Martini R, Schmidt K. 1984. Ultrastructure and early development of the pore plate sensilla of Gymnomerus laevipes (Shuckard) (Vespoidea, Eumenidae). Protoplasma 119:197-211.

Meng LZ, Wu CH, Wicklein M, Kaissling KE, Bestmann HJ. 1989. Number and sensitivity of 3 types of pheromone receptor-cells in Antheraea pernyi and Antheraea polyphemus. J Comp Physiol A:139-146.

Mobbs PG. 1982. The brain of the honeybee Apis mellifera. 1. The connections and spatial-organization of the mushroom bodies. Philos R Soc B 298:309-354

O'Connell RJ, Beuchamp JT, Grant AJ. 1986. Insect olfactory receptor responses to components of pheromone blends. J Chem Ecol 12:451467.

Ochieng SA, Anderson P, Hansson BS. 1995. Antennal lobe projection patterns of olfactory receptor neurons involved in sex-pheromone detection in Spodoptera littoralis (Lepidoptera, Noctuidae). Tissue Cell 27:221-232.

Ochieng SA, Park KC, Zhu JW, Baker TC. 2000. Functional morphology of antennal chemoreceptors of the parasitoid Microplitis croceipes (Hymenoptera: Braconidae). Arthropod Struct Dev 29:231-240.

Ochieng SA, Park KC, Baker TC. 2002. Host plant volatiles synergize responses of sex pheromone-specific olfactory receptor neurons in male Helicoverpa zea. J Comp Physiol A 188:325-333.

Pareto A. 1972. Spatial-distribution of sensory antennal fibers in central nervous-system of worker bees. Z Zellforsch Micr Ann 131:109.

Rospars JP. 1988. Structure and development of the insect antennodeutocerebral system. Int J Insect Morphol Embryol 17:243-294.

Rospars JP, Hildebrand JG. 1992. Anatomical identification of glomeruli in the antennal lobes of the male sphinx moth Manduca sexta. Cell Tissue Res 270:205-227.

Sachse S, Galizia CG. 2002. Role of inhibition for temporal and spatial odor representation in olfactory output neurons: a calcium imaging study. J Neurophysiol 87:1106-1117.

Sachse S, Galizia CG. 2003. The coding of odour-intensity in the honeybee antennal lobe: local computation optimizes odour representation. Eur J Neurosci 18:2119-2132.
Sachse S, Rappert A, Galizia CG. 1999. The spatial representation of chemical structures in the antennal lobe of honeybees: steps towards the olfactory code. Eur J Neurosci 11:3970-3982.

Schneider D, Steinbrecht RA. 1968. Checklist of insect olfactory sensilla. Symp. Zool Soc Lond 23:279-297.

Shanbhag SR, Müller B, Steinbrecht RA. 1999. Atlas of olfactory organs of Drosophila melanogaster 1 . Types, external organization, innervation and distribution of olfactory sensilla. Int J Insect Morphol Embryol $28: 377-397$.

Sigg D, Thompson CM, Mercer AR. 1997. Activity-dependent changes to the brain and behavior of the honey bee, Apis mellifera (L.). J Neurosci 17:7148b-7156.

Steinbrecht R. 1970. Morphometric studies on antenna of silk moth, Bombyx mori $\mathrm{L}-$ number and distribution of olfactory sensilla. Z Morphol Tiere 68:93.

Steiner C, Keil TA. 1995. Morphogenesis of the antenna of the male silkmoth, Antheraea polyphemus .5. Development of the peripheral nervous-system. Tissue Cell 27:275-288.

Stepper J, Becker C, Schmidt K. 1983. Ultrastructure and ontogeny of the pore plates on the antennae of Pimpla turionellae (Hymenoptera, Ichneumonidae). Zoomorphology 102:11-32.

Stocker RF. 1994. The organisation of the chemosensory system in Drosophila melanogaster. Cell Tissue Res 275:3-26.

Stranden M, Liblikas I, Konig WA, Almaas TJ, Borg-Karlson AK, Mustaparta H. 2003. (-)-Germacrene D receptor neurones in three species of heliothine moths: structure-activity relationships. J Comp Physiol A 189:563-577.

Strausfeld NJ, Hildebrand JG. 1999. Olfactory systems: common design, uncommon origins? Curr Opin Neurobiol 9:634-639.

Suzuki H. 1975. Convergence of olfactory inputs from both antennae in brain of honeybee. J Exp Biol 62:11-26.

Vareschi E. 1971. Odor discrimination in honey bee-single cell and behavioral response. Z Vergl Physiol 75:143.

Vermeulen A, Rospars JP. 2004. Why are insect olfactory receptor neurons grouped into sensilla? The teachings of a model investigating the effects of the electrical interaction between neurons on the transepithelial potential and the neuronal transmembrane potential. Eur Biophys J Biophys Lett 33:633-643.

Vickers NJ, Christensen TA, Hildebrand JG. 1998. Combinatorial odor discrimination in the brain: attractive and antagonist odor blends are represented in distinct combinations of uniquely identifable glomeruli. J Comp Neurol 400:35-56.

Vosshall LB, Amrein H, Morozov PS, Rzhetsky A, Axel R. 1999. A spatial map of olfactory receptor expression in the Drosophila antenna. Cell 96:725-736.

Winnington AP, Napper RM, Mercer AR. 1996. Structural plasticity of identified glomeruli in the antennal lobes of the adult worker honey bee. J Comp Neurol 365:479-490. 